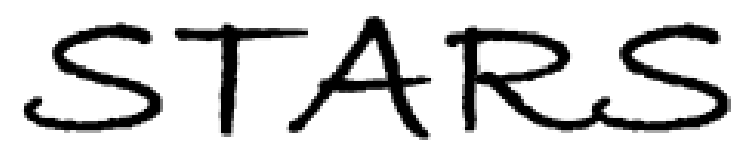

University of Central Florida

STARS

$1-1-1998$

\title{
Power analysis for detecting trends in the presence of concomitant variables
}

David M. Nickerson

University of Central Florida

Arnold Brunell

Find similar works at: https://stars.library.ucf.edu/facultybib1990

University of Central Florida Libraries http://library.ucf.edu

This Article is brought to you for free and open access by the Faculty Bibliography at STARS. It has been accepted for inclusion in Faculty Bibliography 1990s by an authorized administrator of STARS. For more information, please contact STARS@ucf.edu.

\section{Recommended Citation}

Nickerson, David M. and Brunell, Arnold, "Power analysis for detecting trends in the presence of concomitant variables" (1998). Faculty Bibliography 1990s. 2387.

https://stars.library.ucf.edu/facultybib1990/2387

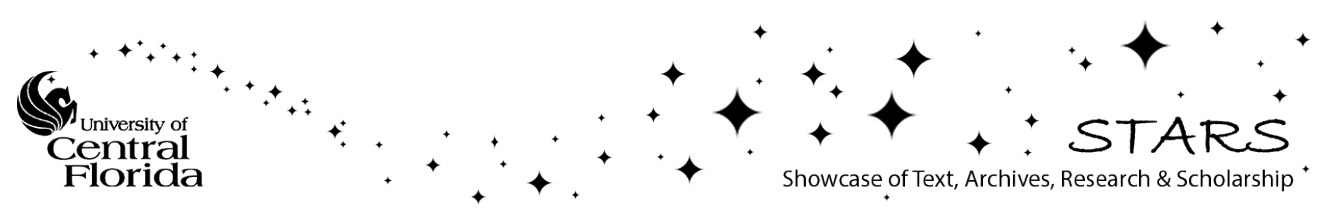




\title{
POWER ANALYSIS FOR DETECTING TRENDS IN THE PRESENCE OF CONCOMITANT VARIABLES
}

\author{
DAVID M. Nickerson ${ }^{1}$ AND ARNOLd BRUnell ${ }^{2}$ \\ ${ }^{1}$ Department of Statistics, University of Central Florida, Orlando, Florida 32816-2370 USA \\ ${ }^{2}$ Florida Game and Fresh Water Fish Commission, 601 West Woodward Avenue, Eustis, Florida 32726 USA
}

\begin{abstract}
In some situations, survey counts must be adjusted for the effects of concomitant variables using analysis of covariance in order to detect a trend in abundance over time. A power analysis in these cases allows the calculation of the probability of detecting a trend after adjustments. The methods will be applicable to samples taken at regular or irregular intervals in time or space, and in which the effects of the concomitant variables do not change over time. Furthermore, the detection of linear and intrinsically linear change can be determined. The methods are applied to the monitoring of American alligator ( $A l$ ligator mississippiensis) populations in Florida.
\end{abstract}

Key words: adjusted means; Alligator mississippiensis; analysis of covariance; weighted least squares estimators.

\section{INTRODUCTION}

Wildlife management often requires the detection of trends in abundance over time, after adjusting survey counts for concomitant variables that are known to affect observability (e.g., rainfall, temperature, etc.). Here, as in Gerrodette $(1987,1991)$ and Link and Hatfield (1990), we wish to determine whether counts are increasing or decreasing over time using a straight line regression. In some applications (e.g., a power analysis) it is unnecessary to precisely model the pattern of change. In these cases, it is sufficient to determine whether there is, over time, a general propensity for the counts to become greater or less than their initial levels. This can be easily and sufficiently accomplished by modeling the counts after adjustments with a simple linear regression over time.

In this article we shall consider designing a sampling scheme for the detection of trends in abundance in the presence of concomitant variables. In this case, there are several questions that can be addressed. For instance, one question of interest is, How much data do we collect to be reasonably certain that we declare significantly different from 0 , a trend of a given magnitude? Another question of interest would be, For a given sampling intensity what magnitude of trend would we be reasonably certain to declare significant? To answer these questions sufficiently, an a priori power analysis must be conducted.

The objectives of this article are similar to those of Gerrodette (1987, 1991) and Link and Hatfield (1990). Here, however, we extend the power analysis to the case of trends in counts adjusted for concomitant variables as in the analysis of covariance (see, e.g., Cochran 1957 for a general discussion of analysis of covari-

Manuscript received 19 April 1996; revised 12 May 1997; accepted 17 May 1997. ance). Specifically, we will exhibit the necessary computations of the probability of declaring significantly different from 0 trends of a given magnitude after adjustment for the effects of concomitant variables for a given amount of sampling.

\section{Methods}

Let us consider the usual set-up in analysis of covariance for the one-way classification with $k(\geq 1)$ covariates or concomitant variables. Here, $t(\geq 2)$ may represent the number of years in which data are collected on abundance and the $k$ covariates. Within the $i$ th year we have $n_{i}$ observations on abundance $\left(Y_{i j}\right)$ and the $k$ covariates $\left(X_{i j 1}, X_{i j 2}, \ldots, X_{i j k}\right), j=1,2, \ldots, n_{i}$ with $i$ $=1,2, \ldots, t$. Here, the $n_{i}$ 's are not necessarily equal and we require $n_{i} \geq 2$ for at least one year. We are assuming that we have included all the covariates that affect observed counts (but not population size), being careful to eliminate those that are unnecessary. Of the two actions, the greater danger is the exclusion of those covariates which are necessary.

As a matter of convenience (see, e.g., Cochran 1957), we will require for the $m$ th covariate, $m=1,2, \ldots, k$,

$$
\sum_{i=1}^{t} \sum_{j=1}^{n_{i}} X_{i j m}=0
$$

This can be easily achieved by redefining each covariate as

$$
X_{i j m}^{*}=X_{i j m}-\bar{X}_{m}
$$

where

$$
\bar{X}_{m}=n^{-1} \sum_{i=1}^{t} \sum_{j=1}^{n_{i}} X_{i j m}
$$

and 


$$
n=\sum_{i=1}^{t} n_{i}
$$

Consider now the usual analysis of covariance model

$$
Y_{i j}=\mu_{i}+\beta_{1} X_{i j 1}+\beta_{2} X_{i j 2}+\ldots+\beta_{k} X_{i j k}+\varepsilon_{i j}
$$

where the $\varepsilon_{i j}$ 's are independent and identically distributed (i.i.d.) normal random variables with mean 0 and variance $\sigma^{2}, i=1,2, \ldots, t$ and $j=1,2, \ldots, n_{i}$. Here, we are assuming that the effects of the covariates on observed counts are additive and can be sufficiently accounted for by linear terms in Eq. 1. Also, the $\mu_{i}$ 's are the mean counts and are generally only indices to abundance.

It is important to note that correct specification of the functional form of the effects of the covariates is critical. Incorrect specification can lead to serious bias in the estimates of $\mu_{1}, \mu_{2}, \ldots, \mu_{t}$ and invalidate the analysis.

In a typical analysis of covariance, one of the major objectives is to estimate the mean count for each year, adjusting for the effects of the $k$ covariates in the given year. Given Eq. 1, these adjusted means are simply the least squares estimates of $\mu_{1}, \mu_{2}, \ldots, \mu_{t}$ obtained simultaneously with the estimates of $\beta_{1}, \beta_{2}, \ldots, \beta_{k}$, the effects of the covariates.

Eq. 1 can be written as

$$
\begin{aligned}
Y_{i j}= & \mu_{1} Z_{i j 1}+\mu_{2} Z_{i j 2}+\ldots+\mu_{t} Z_{i j t}+\beta_{1} X_{i j 1} \\
& +\beta_{2} X_{i j 2}+\ldots+\beta_{k} X_{i j k}+\varepsilon_{i j}
\end{aligned}
$$

where

$$
\begin{aligned}
Z_{i j m} & =1, \quad \text { if } i=m \\
& =0, \quad \text { if } i \neq m
\end{aligned}
$$

$i=1,2, \ldots, t, j=1,2, \ldots, n_{i}$ and $m=1,2, \ldots, t$. Consequently, in matrix notation, Eq. 2 becomes

$$
\mathbf{Y}=\mathbf{Z} \mu+\mathbf{X} \beta+\varepsilon=[\mathbf{Z X}]\left[\begin{array}{l}
\mu \\
\beta
\end{array}\right]+\varepsilon
$$

where $\mathbf{Y}=\left[\mathbf{Y}_{1}{ }^{\prime}, \mathbf{Y}_{2}{ }^{\prime}, \ldots, \mathbf{Y}_{t}{ }^{\prime}\right]^{\prime}$ with $\mathbf{Y}_{i}=\left[Y_{i 1}, Y_{i 2}\right.$, $\left.\ldots, Y_{i n_{i}}\right]^{\prime}, \mu=\left[\mu_{1}, \mu_{2}, \ldots, \mu_{t}\right]^{\prime}, \beta=\left[\beta_{1}, \beta_{2}, \ldots\right.$, $\left.\beta_{k}\right]^{\prime}, \varepsilon=\left[\varepsilon_{1}{ }^{\prime}, \varepsilon_{2}{ }^{\prime}, \ldots, \varepsilon_{t}{ }^{\prime}\right]^{\prime}$ with $\varepsilon_{i}=\left[\varepsilon_{i 1}, \varepsilon_{i 2}, \ldots\right.$, $\left.\varepsilon_{i n_{i}}\right]^{\prime}, \mathbf{Z}=\left[\mathbf{Z}_{1}{ }^{\prime}, \mathbf{Z}_{2}{ }^{\prime}, \ldots, \mathbf{Z}_{t}{ }^{\prime}\right]^{\prime}$ with

$$
\mathbf{Z}_{i}=\left[\begin{array}{cccc}
Z_{i 11} & Z_{i 12} & \cdots & Z_{i 1 t} \\
Z_{i 21} & Z_{i 22} & \cdots & Z_{i 2 t} \\
\vdots & \vdots & & \vdots \\
Z_{i n_{i} 1} & Z_{i n_{i} 2} & \cdots & Z_{i n_{i}}
\end{array}\right]
$$

and $\mathbf{X}=\left[\mathbf{X}_{1}{ }^{\prime}, \mathbf{X}_{2}{ }^{\prime}, \ldots, \mathbf{X}_{t}{ }^{\prime}\right]^{\prime}$ with

$$
\mathbf{X}_{i}=\left[\begin{array}{cccc}
X_{i 11} & X_{i 12} & \cdots & X_{i 1 k} \\
X_{i 21} & X_{i 22} & \cdots & X_{i 2 k} \\
\vdots & \vdots & & \vdots \\
X_{i n_{i} 1} & X_{i n_{i 2}} & \cdots & X_{i n_{i} k}
\end{array}\right] .
$$

Given the assumptions on $\varepsilon$, the maximum likelihood estimators can be obtained by least squares (LS). These are

$$
\begin{aligned}
{\left[\begin{array}{l}
\mathbf{m} \\
\mathbf{b}
\end{array}\right] } & =\left[\begin{array}{ll}
\mathbf{Z}^{\prime} \mathbf{Z} & \mathbf{Z}^{\prime} \mathbf{X} \\
\mathbf{X}^{\prime} \mathbf{Z} & \mathbf{X}^{\prime} \mathbf{X}
\end{array}\right]^{-1}\left[\begin{array}{l}
\mathbf{Z}^{\prime} \mathbf{Y} \\
\mathbf{X}^{\prime} \mathbf{Y}
\end{array}\right] \\
& =\left[\begin{array}{cc}
\mathbf{W} & -\mathbf{W B D}^{-1} \\
-\mathbf{D}^{-1} \mathbf{B}^{\prime} \mathbf{W} & \mathbf{D}^{-1}+\mathbf{D}^{-1} \mathbf{B}^{\prime} \mathbf{W} \mathbf{B} \mathbf{D}^{-1}
\end{array}\right]\left[\begin{array}{l}
\mathbf{Z}^{\prime} \mathbf{Y} \\
\mathbf{X}^{\prime} \mathbf{Y}
\end{array}\right]
\end{aligned}
$$

where $\mathbf{A}=\mathbf{Z}^{\prime} \mathbf{Z}, \mathbf{B}=\mathbf{Z}^{\prime} \mathbf{X}, \mathbf{D}=\mathbf{X}^{\prime} \mathbf{X}$, and $\mathbf{W}=(\mathbf{A}-$ $\left.\mathbf{B D}^{-1} \mathbf{B}^{\prime}\right)^{-1}$. Therefore, the vector of adjusted count means is given by

$$
\begin{aligned}
\mathbf{m} & =\mathbf{W Z} \mathbf{Z}^{\prime} \mathbf{Y}-\mathbf{W B D}^{-1} \mathbf{X}^{\prime} \mathbf{Y} \\
& =\mathbf{W}\left(\mathbf{Z}-\mathbf{X D}^{-1} \mathbf{B}^{\prime}\right)^{\prime} \mathbf{Y}=\left(\mathbf{Z}^{\prime} \mathbf{P Z}\right)^{-1} \mathbf{Z}^{\prime} \mathbf{P Y}
\end{aligned}
$$

where $\mathbf{P}=\mathbf{I}-\mathbf{X}\left(\mathbf{X}^{\prime} \mathbf{X}\right)^{-1} \mathbf{X}^{\prime}$. Furthermore, under the normality assumption on $\varepsilon$, we have

$$
\mathbf{m} \sim \mathrm{N}\left(\mu, \sigma^{2}\left(\mathbf{Z}^{\prime} \mathbf{P Z}\right)^{-1}\right)
$$

i.e., $\mathbf{m}$ has a multivariate normal distribution with mean vector $\mu$ and covariance matrix $\sigma^{2}\left(\mathbf{Z}^{\prime} \mathbf{P Z}\right)^{-1}$.

Consider now the objective of estimation and testing of a linear trend in the mean counts across years. With this end, let us define $c_{i}$ as the year value for the $i$ th year, $i=1,2, \ldots, t$. Typically $c_{i}=i$. However, if there are years in which data were not collected this may not be the case. For instance, for the 1 st yr of data collection $c_{1}=1$. If, however, the 2 nd $\mathrm{yr}$ of data collection occurred 2 yr later, then $c_{2}=3$, etc. Here, we wish to characterize the linear trend among the mean counts as

$$
\mu_{i}=\Gamma_{0}+\Gamma_{1} c_{i}
$$

where $i=1,2, \ldots, t$. This model implies that the mean counts fall precisely on a straight line and that $\Gamma_{1}$ represents the direction and magnitude of the linear trend. An alternative approach would be to forgo the restriction given in Eq. 4, fit a straight line directly to the $\mu_{i}$ 's, and define trend as the slope of this line.

In matrix terms, let us write Eq. 4 as

$$
\mu=\mathbf{C} \Gamma
$$

where $\mu$ is defined as before,

$$
\mathbf{C}=\left[\begin{array}{cccc}
1 & 1 & \cdots & 1 \\
c_{1} & c_{2} & \cdots & c_{t}
\end{array}\right]^{\prime}
$$

and

$$
\Gamma=\left[\begin{array}{c}
\Gamma_{0} \\
\Gamma_{1}
\end{array}\right] .
$$

Since $\mathbf{m}$, our estimator of $\mu$, has a covariance matrix that is not a constant times the identity matrix, we must resort to a weighted least squares (WLS) approach to obtain the most efficient estimator for $\Gamma$ (the trend line), and in particular $\Gamma_{1}$ (the trend). This type of estimator is a slight deviation from Gerrodette's least squares (LS) estimator. However, the WLS estimator is better 
in every sense (except simplicity) than the LS estimator for this situation. Using well-established results (see, e.g., Neter et al. 1990, pp. 418-424), we have as the WLS estimator of $\Gamma$,

$$
\mathbf{g}=\left(\mathbf{C}^{\prime}\left(\mathbf{Z}^{\prime} \mathbf{P Z}\right) \mathbf{C}\right)^{-1} \mathbf{C}^{\prime}\left(\mathbf{Z}^{\prime} \mathbf{P Z}\right) \mathbf{m} .
$$

Here, tests of significance can be based on

$$
\mathbf{g} \sim N\left\{\Gamma, \sigma^{2}\left[\mathbf{C}^{\prime}\left(\mathbf{Z}^{\prime} \mathbf{P Z}\right) \mathbf{C}\right]^{-1}\right\} .
$$

In particular, if we believe that Eq. 4 is true, then from Eq. 7, to test whether a linear trend in the adjusted counts is present, i.e., to test

$$
H_{0}: \Gamma_{1}=0 \text { vs. } H_{\mathrm{a}}: \Gamma_{1} \neq 0
$$

we have as our test statistic

$$
t=g_{1} / s\left(g_{1}\right)
$$

where

$$
s^{2}\left(g_{1}\right)=\operatorname{MSE}\left[\mathbf{C}^{\prime}\left(\mathbf{Z}^{\prime} \mathbf{P Z}\right) \mathbf{C}\right]^{22}
$$

with $g_{1}$ the second element of $\mathbf{g}, \operatorname{MSE}=\operatorname{SSE} /[n-(k+$ 2)],

$$
\operatorname{SSE}=\mathbf{Y}^{\prime}\left(\mathbf{I}-[\mathbf{Z C X}]\left[\begin{array}{cc}
\mathbf{C}^{\prime} \mathbf{Z}^{\prime} \mathbf{Z C} & \mathbf{C}^{\prime} \mathbf{Z}^{\prime} \mathbf{X} \\
\mathbf{X}^{\prime} \mathbf{Z C} & \mathbf{X}^{\prime} \mathbf{X}
\end{array}\right]^{-1}\left[\begin{array}{c}
\mathbf{C}^{\prime} \mathbf{Z}^{\prime} \\
\mathbf{X}^{\prime}
\end{array}\right]\right) \mathbf{Y}
$$

and $\left[\mathbf{C}^{\prime}\left(\mathbf{Z}^{\prime} \mathbf{P Z}\right) \mathbf{C}\right]^{22}$ the last diagonal element of $\left[\mathbf{C}^{\prime}\left(\mathbf{Z}^{\prime} \mathbf{P Z}\right) \mathbf{C}\right]^{-1}$. Note that SSE is the sum of squared errors from fitting the model in Eq. 1 under the restriction given in Eq. 4, i.e.,

$$
Y_{i j}=\Gamma_{0}+\Gamma_{1} c_{i}+\beta_{1} X_{i j 1}+\beta_{2} X_{i j 2}+\ldots+\beta_{k} X_{i j k}+\varepsilon_{i j} .
$$

A significant linear trend is declared at the $\alpha$ level of significance if

$$
|t|>t(1-\alpha / 2 ; n-[k+2])
$$

where $t(1-\alpha / 2 ; n-[k+2])$ is the $(1-\alpha / 2)$ th percentile of the $t$ distribution with $[n-(k+2)]$ degrees of freedom.

Based on the $t$ statistic the probability of declaring a trend significantly different from 0 (i.e., the power of the test) is given by

$$
\text { power }=\mathrm{P}[|t(d f, \theta)|>t(1-\alpha / 2 ; d f)]
$$

where $t(d f, \theta)$ is distributed as a noncentral $t$ with [ $n$ $-(k+2)$ ] degrees of freedom, and noncentrality parameter

$$
\theta=\Gamma_{1} / \sigma\left(g_{1}\right)
$$

with

$$
\sigma^{2}\left(g_{1}\right)=\sigma^{2}\left[\mathbf{C}^{\prime}\left(\mathbf{Z}^{\prime} \mathbf{P Z}\right) \mathbf{C}\right]^{22} .
$$

Consequently the power calculations depend on only $\alpha, d f$, and $\theta$. However, $\theta$ depends on $\Gamma_{1}, \sigma^{2}$, and $\left[\mathbf{C}^{\prime}\left(\mathbf{Z}^{\prime} \mathbf{P Z}\right) \mathbf{C}\right]^{22}$ where

$$
\left[\mathbf{C}^{\prime}\left(\mathbf{Z}^{\prime} \mathbf{P Z}\right) \mathbf{C}\right]^{22}=\left[\left(1-R^{2}\right) \sum_{i=1}^{t} n_{i}\left(c_{i}-\bar{c}\right)^{2}\right]^{-1}
$$

with

$$
\bar{c}=n^{-1} \sum_{i=1}^{t} n_{i} c_{i}
$$

and $R^{2}$ is the coefficient of multiple determination of year with $X_{1}, X_{2}, \ldots, X_{k}$. Therefore, the power calculations depend on the values of the concomitant variables only through their squared multiple correlation with year.

At this point let us make two simplifying assumptions. First, we assume that the number of observations within a year is the same for each year (i.e., $n_{i}=n_{0}$ ). Second, the $t$ years are consecutive. With these two assumptions, following Gerrodette (1987),

$$
\sum_{i=1}^{t} n_{i}\left(c_{i}-\bar{c}\right)^{2}=n_{0} t\left(t^{2}-1\right) / 12 .
$$

Consequently, from Eqs. 10, 11, and 12,

$$
\theta=\sqrt{n_{0} \frac{t\left(t^{2}-1\right)}{12}\left(1-R^{2}\right)} \frac{\Gamma_{1}}{\sigma} .
$$

Therefore, assuming that $\sigma^{2}$ and $R^{2}$ are estimated from previous studies, the choices the experimenter has in power calculations are the values for $\alpha, \Gamma_{1}, n_{0}$, and $t$.

In a typical power analysis of this sort, the researcher will fix $\alpha$ and either fix $n_{0}$ and $t$ and vary $\Gamma_{1}$, or fix $\Gamma_{1}$ and vary $n_{0}$ and $t$. In each specification, the power is computed and a plot of power vs. varied parameter is obtained.

\section{RESUlts}

In this section, the methods detailed above are applied to an actual data set. The sample data are from night spotlight surveys of alligator populations in north Florida. The complete data set is divided into transects where within each transect there can be several observations per year for the years 1977-1993. Furthermore, each transect might not have data for every year in the study. Each observation consists of alligator counts per size class, water level, and water temperature.

One objective of the study was to determine whether there was an increase or decrease over time in the counts for $122-\mathrm{cm}$ and longer alligators. However, as noted in Woodward and Marion (1978) and Woodward and Moore (1990), factors such as water level and water temperature affect the observed counts. Here, the effect of water level and temperature is on the ability to observe the alligators, not on the actual number of alligators present. Consequently, adjustments to the observed counts within a year must be made prior to fitting a trend to the adjusted counts.

A particular transect (Lake George) was chosen to demonstrate the power calculations. Table 1 contains the observations for this transect.

For year $i=1,2, \ldots, 6$ and survey $j=1,2, \ldots$, $n_{i}$ within year $i$, the model in the form of Eq. 1 fit to this data was 
TABLE 1. Observed water temperature, water level (meters above mean sea level), and count of 122-cm and longer alligators on Lake George for the years 1985-1990.

\begin{tabular}{|c|c|c|c|}
\hline Year & $\begin{array}{c}\text { Water } \\
\text { temperature } \\
\left({ }^{\circ} \mathrm{C}\right)\end{array}$ & Water level & Count \\
\hline 1985 & 28 & 0 & 317 \\
\hline 1986 & 29 & 0.3 & 295 \\
\hline 1986 & 29 & 0 & 336 \\
\hline 1987 & 28 & 0 & 241 \\
\hline 1987 & 30 & 0 & 321 \\
\hline 1988 & 26 & 0.3 & 184 \\
\hline 1988 & 28 & 0.3 & 286 \\
\hline 1989 & 28 & 0 & 304 \\
\hline 1989 & 30 & 0.3 & 288 \\
\hline 1990 & 31 & 0 & 245 \\
\hline 1990 & 32 & 0.3 & 296 \\
\hline
\end{tabular}

$$
Y_{i j}=\mu_{i}+\beta_{1} X_{i j 1}+\beta_{2} X_{i j 2}+\varepsilon_{i j}
$$

where

$$
Y_{i j}=\text { number of 122-cm and longer alligators for }
$$

$j$ th survey in $i$ th year

$X_{i j 1}=$ [water temperature for $j$ th survey in $i$ th year] $-\bar{X}_{1}$

and

$$
\left.X_{i j 2}=\text { [water level for } j \text { th survey in } i \text { th year }\right]-\bar{X}_{2}
$$

with $\bar{X}_{1}=$ mean water temperature and $\bar{X}_{2}=$ mean water level. lows

By Eq. 2, our input vectors and matrices are as fol-

$$
\begin{aligned}
& \mathbf{Y}=\left[\begin{array}{l}
317 \\
295 \\
336 \\
241 \\
321 \\
184 \\
286 \\
304 \\
288 \\
245 \\
296
\end{array}\right] \quad \mathbf{Z}=\left[\begin{array}{llllll}
1 & 0 & 0 & 0 & 0 & 0 \\
0 & 1 & 0 & 0 & 0 & 0 \\
0 & 1 & 0 & 0 & 0 & 0 \\
0 & 0 & 1 & 0 & 0 & 0 \\
0 & 0 & 1 & 0 & 0 & 0 \\
0 & 0 & 0 & 1 & 0 & 0 \\
0 & 0 & 0 & 1 & 0 & 0 \\
0 & 0 & 0 & 0 & 1 & 0 \\
0 & 0 & 0 & 0 & 1 & 0 \\
0 & 0 & 0 & 0 & 0 & 1 \\
0 & 0 & 0 & 0 & 0 & 1
\end{array}\right] \quad \mathbf{X}=\left[\begin{array}{cr}
-1 & -0.136 \\
0 & 0.164 \\
0 & -0.136 \\
-1 & -0.136 \\
-1 & -0.136 \\
-1 & 0.164 \\
-1 & -0.136 \\
1 & 0.164 \\
2 & -0.136 \\
3 & 0.164
\end{array}\right] \\
& \mu=\left[\mu_{1} \mu_{2} \mu_{3} \mu_{4} \mu_{5} \mu_{6}\right]^{\prime}
\end{aligned}
$$

and

$$
\beta=\left[\beta_{1} \beta_{2}\right]^{\prime} .
$$

Consequently, by Eq. 3, the adjusted mean alligator counts are

$\mathbf{m}=\left[\begin{array}{llllll}337.3 & 317.4 & 262.4 & 335.1 & 297.9 & 175.1\end{array}\right]^{\prime}$.

Fig. 1 is a plot of the unadjusted and adjusted mean alligator counts over time. From Fig. 1, there appears to be a decreasing trend in the adjusted counts over the

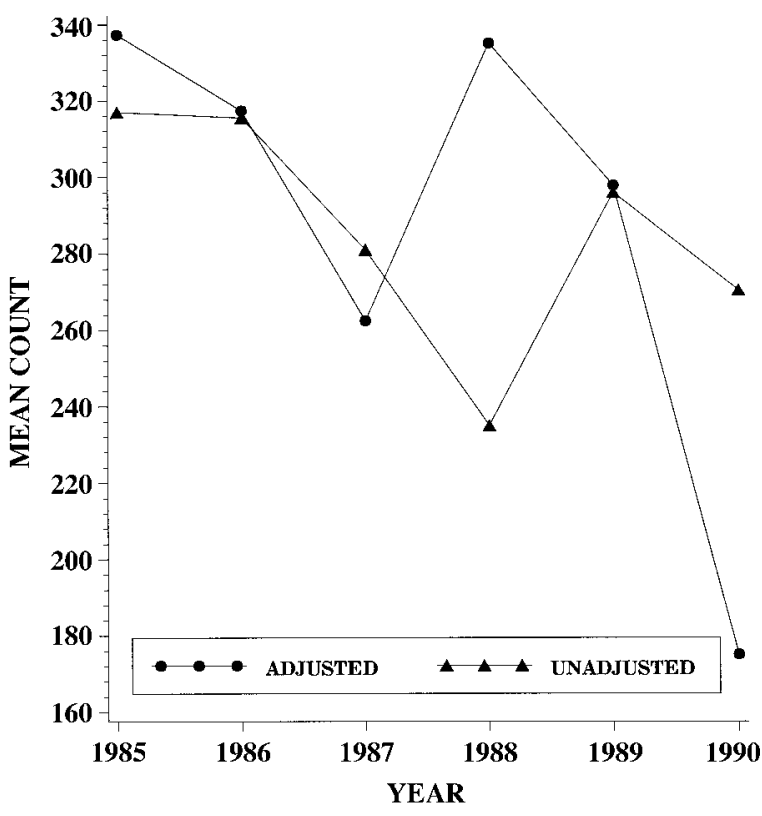

FIG. 1. Adjusted and unadjusted mean alligator counts vs. time on Lake George for $122-\mathrm{cm}$ and longer alligators.

observed years. However, to formally test this conjecture we shall follow the procedure outlined in the previous section.

By Eq. 5, we have

$$
\mathbf{C}=\left[\begin{array}{llllll}
1 & 1 & 1 & 1 & 1 & 1 \\
1 & 2 & 3 & 4 & 5 & 6
\end{array}\right]^{\prime}
$$

and hence by Eq. 6, the estimate for $\Gamma$ is

$$
\mathbf{g}=[343.84-16.32]^{\prime} .
$$

Consequently, from $\mathbf{g}$ we estimate the annual change in number of alligators to be -16.32 (i.e., a decrease of $\sim 16$ alligators per year).

To formally test whether there is a significant linear trend in the adjusted counts, i.e., to test

$$
H_{0}: \Gamma_{1}=0 \quad \text { vs. } H_{\mathrm{a}}: \Gamma_{1} \neq 0
$$

we have by Eq. 8

$$
t=\frac{-16.32}{8.136}=-2.006
$$

with rejection region (at $\alpha=0.05$ )

$$
|t|>t(0.975 ; 7)=2.365 \text {. }
$$

Therefore, at the 0.05 significance level $(P$ value $=$ 0.0848 ) we cannot conclude that there is a linear trend in the adjusted counts over time.

This leads us to the power analysis. We may now ask questions concerning future studies on this transect or transects with similar characteristics, i.e., transects with an $R^{2}$ (the coefficient of multiple determination of year with water temperature and level) of $\sim 0.2869$ and a $\sigma^{2}$ of $\sim 1330.34$. 


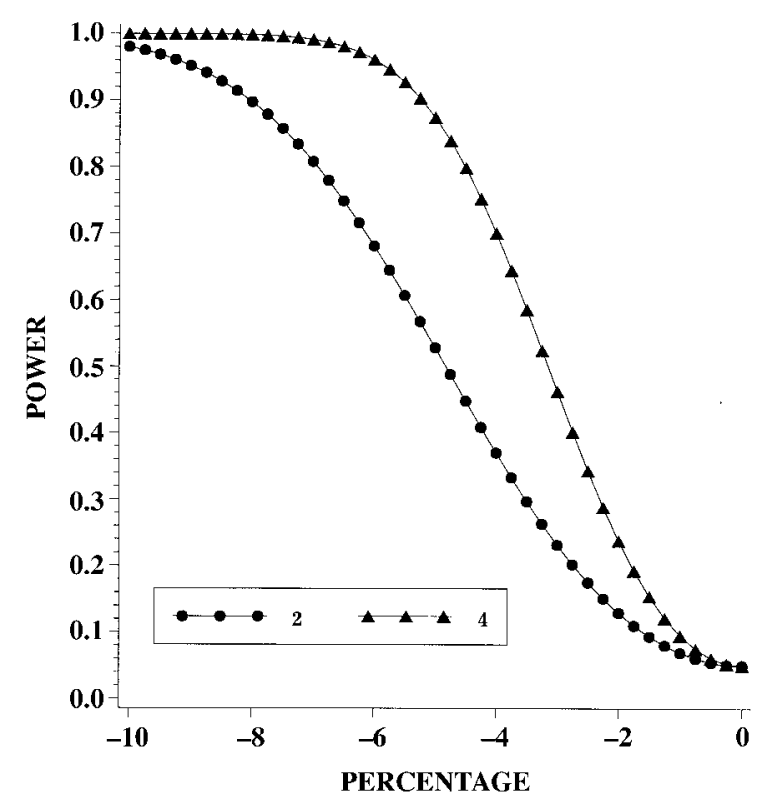

FIG. 2. Power of simple linear regression of adjusted mean counts over time for Lake George for $122-\mathrm{cm}$ and longer alligators. Curves show the relationship of power to $100 \mathrm{r} \%$ for $n_{0}=2$ and $4, t=6$, and $\alpha=0.05$, where $337.3 r$ is the assumed yearly increase.

Using the adjusted count for the 1st yr (1985) as the baseline (i.e., $m_{1}=337.3$ ) we can compute power of detecting a linear trend of $\Gamma_{1}=r(337.3)$ for various values of $r$. Furthermore, the Florida Game and Fresh Water Fish Commission (Commission), which conducted the surveys, was mainly interested in detecting a yearly decrease in the counts of $122-\mathrm{cm}$ and longer alligators. This would indicate a possible decline in abundance on the particular transect in question and would allow the commission to take appropriate action. Consequently, we considered values of $r<0$.

We now consider obtaining additional data sets with various values of $n_{0}$ and $t$. For each combination of $n_{0}$ and $t$, we can obtain the power of detecting a trend of magnitude $\Gamma_{1}$. In this way, we can determine what sampling intensity is required to declare a linear trend significant for a given magnitude. Also, we can determine what magnitude of trend is required to be declared significant for a given sampling intensity. Fig. 2 is a plot of power vs $100 r \%$ for $t=6, n_{0}=2$ and 4 , and $\alpha=0.05$, while Fig. 3 is a plot of power vs. $t$ for $n_{0}$ $=2,100 r \%=-2.5$ and $-5 \%$, and $\alpha=0.05$.

Recall that the estimated slope in the adjusted counts from the original data was $g_{1}=-16.32$. This translates into $100 r \%=100(-16.32 / 337.3) \%=-4.8 \%$. Consequently, from Fig. 2, if we sample the same number of years, $t=6$, but sample $n_{0}=4$ times per year (instead of $\sim 2$ ) and if the actual decrease is 16.32 alligators per year as estimated, then we will have an $84 \%$ chance of claiming that the trend is significantly different from 0 . Consequently, future studies of $6 \mathrm{yr}$

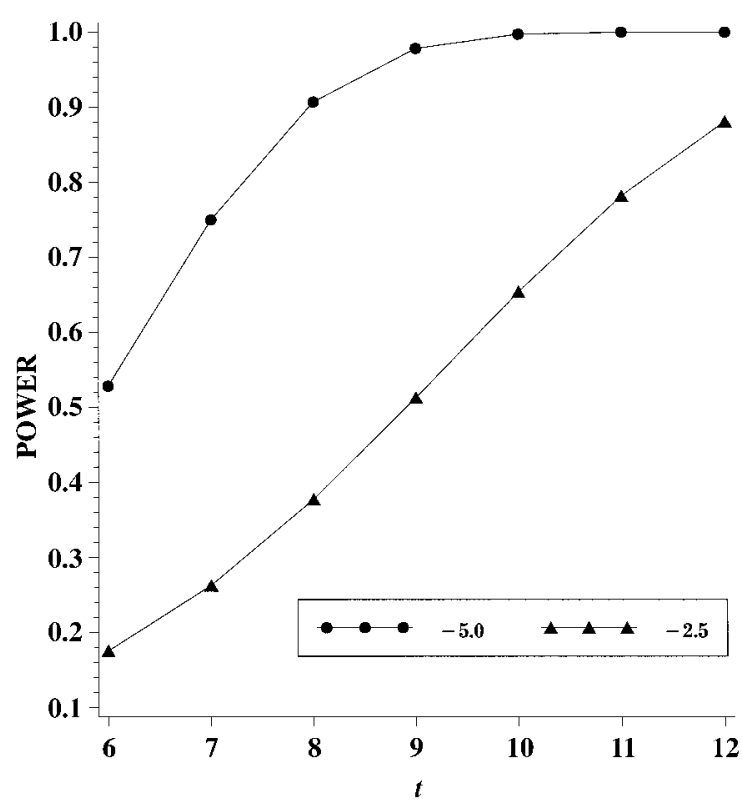

FIG. 3. Power of simple linear regression of adjusted mean counts over time for Lake George for $122-\mathrm{cm}$ and longer alligators. Curves show the relationship of power to $t$ for $100 r \%=-2.5$ and $-5, n_{0}=2$, and $\alpha=0.05$, where $337.3 r$ is the assumed yearly increase.

in length on this or similar transects with an actual decline of 16.32 alligators per year should be sampled 4 times per year to have high power in detecting a significant trend.

Likewise, from Fig. 3, if we sample for $t=8 \mathrm{yr}$ (instead of 6) and sample approximately the same number of times per year, $n_{0}=2$, we will have over a $90 \%$ chance of claiming a significant trend if the actual decrease is $5 \%$ per year. Consequently, future studies with two samples per year on this or similar transects with an actual decline of 5\% (of 337.3) per year should be sampled for $8 \mathrm{yr}$ to have high power in detecting a significant trend.

\section{DISCUSSION}

This paper considers the computation of power for detecting trends in counts after adjusting for concomitant variables. This differs from the work of Gerrodette (1987, 1991) and Link and Hatfield (1990) in that they do not consider the computation of power in the presence of concomitant variables. As in many biological applications, observed counts are affected by extraneous variables. As a consequence, the observed counts must be adjusted for the effects of these variables. However, if in the current setting the $n_{i}$ 's were all equal and the yearly average of each concomitant variable did not vary from year to year, the problem would reduce to Gerrodette's homoscedastic model (see the Appendix in Link and Hatfield [1990]). 


\section{ACKNOWLEDGMENTS}

The authors are grateful to Steve Linda for his comments on a preliminary draft of the manuscript. The authors are also grateful to the referees for their thorough review.

\section{Literature Cited}

Cochran, W. G. 1957. Analysis of covariance: its nature and uses. Biometrics 13:261-281.

Gerrodette, T. 1987. A power analysis for detecting trends. Ecology 68:1364-1372.

. 1991. Models for power of detecting trends - a reply to Link and Hatfield. Ecology 72:1889-1892.
Link, W. A., and J. S. Hatfield. 1990. Power calculations and model selection for trend analysis: a comment. Ecology 71:1217-1220.

Neter, J., W. Wasserman, and M. H. Kutner. 1990. Applied linear statistical models. Third edition. Richard D. Irwin, Boston, Massachusetts, USA.

Woodward, A. R., and W. R. Marion. 1978. An evaluation of factors affecting night-light counts of alligators. Proceedings of the Annual Conference of the Southeast Association of Fish and Wildlife Agencies 32:291-302.

Woodward, A. R., and C. T. Moore. 1990. Statewide alligator surveys. Final Performance Report. Florida Game and Fresh Water Fish Commission, Tallahassee, Florida, USA. 